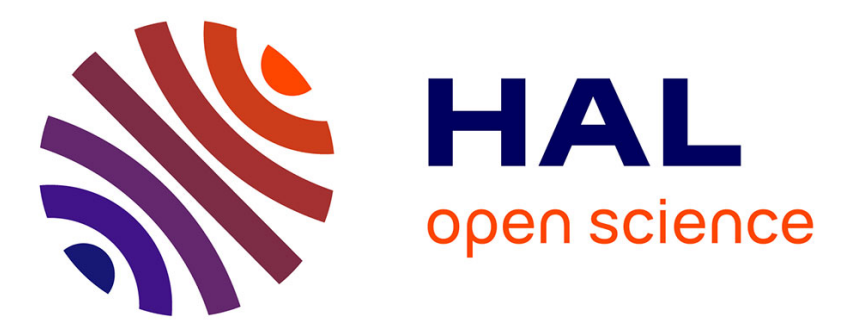

\title{
Transfert de la conscience morphologique de l'arabe langue première au français langue seconde au cours des trois premières années d'apprentissage
}

Anne-Sophie Besse, N. Marec-Breton, Carolina Ferreira, J.-E. Gombert

\section{- To cite this version:}

Anne-Sophie Besse, N. Marec-Breton, Carolina Ferreira, J.-E. Gombert. Transfert de la conscience morphologique de l'arabe langue première au français langue seconde au cours des trois premières années d'apprentissage. Psychologie Française, 2019, 64 (1), pp.85-102. 10.1016/j.psfr.2018.05.001 . hal-02935004

\section{HAL Id: hal-02935004 \\ https://hal.science/hal-02935004}

Submitted on 9 Sep 2020

HAL is a multi-disciplinary open access archive for the deposit and dissemination of scientific research documents, whether they are published or not. The documents may come from teaching and research institutions in France or abroad, or from public or private research centers.
L'archive ouverte pluridisciplinaire HAL, est destinée au dépôt et à la diffusion de documents scientifiques de niveau recherche, publiés ou non, émanant des établissements d'enseignement et de recherche français ou étrangers, des laboratoires publics ou privés. 
Transfert de la conscience morphologique de l'arabe langue première au français langue seconde au cours des trois premières années d'apprentissage

Transfer of morphological awareness between Arabic first language and French second language during the first three years of learning

A.-S. Besse ${ }^{\mathrm{a}, *, 1}$, N. Marec-Breton ${ }^{\mathrm{b}, 2}$,

C. Roganti Leite Moreira ${ }^{\mathrm{a}, 3}$, J.-E. Gombert ${ }^{\mathrm{c}, 4}$

a Laboratoire psychologie des cognitions (LPC, EA 4440), 12, rue Goethe, 67000 Strasbourg, France

${ }^{\mathrm{b}}$ Centre de recherches en psychologie, cognition et communication (CRPCC, EA 1285), université Rennes 2,

place du recteur-Henri-le-Moal, 35043 Rennes cedex, France

c CRPCC EA1285, université Rennes 2, place du recteur-Henri-Le-Moal, CS 24307, 35043 Rennes cedex,

France

i n fo a r t i c 1 e

Historique de l'article:

Reçu le 27 avril 2016

Accepté le 2 mai 2018

\section{$r$ é s u m é}

Cette étude examine le transfert de la conscience morphologique (CM) de 1'arabe L1 vers la CM et la lecture de mots en français L2 $\left(\mathrm{F}_{\mathrm{L} 2}\right)$ en $1^{\mathrm{e}}, 2^{\mathrm{e}}$ et $3^{\mathrm{e}}$ années d'apprentissage du $\mathrm{F}_{\mathrm{L} 2}(\mathrm{n}=106)$. Les niveaux de lecture et de $\mathrm{CM}$ flexionnelle et dérivationnelle sont

\footnotetext{
* Auteur correspondant.

Adresse e-mail : anne-sophie.besse@unistra.fr (A.-S. Besse).

Thèmes de recherche : apprentissage de la lecture, bilinguisme, apprentissage d'une langue seconde, conscience et traitement morphologiques.

2 Thèmes de recherche : apprentissage de la lecture, développement des habiletés métalinguistiques (conscience morphologique), bilinguisme, troubles des apprentissages.

3 Thèmes de recherche : apprentissage de la lecture, conscience morphologique, apprentissage d'une langue seconde, bilinguisme.

${ }^{4}$ Thèmes de recherche : apprentissage de la lecture, développement métalinguistique.
} 
Mots clés :

Transfert interlangue Conscience morphologique Morphologie linéaire/non linéaire

Reconnaissance des mots écrits

Apprentissage en langue seconde mesurés dans les deux langues par des tests de lecture de mots en une minute et des épreuves de détection d'intrus (flexionnel et dérivationnel). Les analyses de régression hiérarchiques montrent que la contribution de la $\mathrm{CM}$ en $\mathrm{L} 1$ à la $\mathrm{CM}$ en L2 n'apparaît qu'en $2^{\mathrm{e}}$ année et sa contribution à la lecture en $\mathrm{F}_{\mathrm{L} 2}$ qu'en $3^{\mathrm{e}}$ année. Le transfert morphologique, notamment dérivationnel, entre des langues orthographiquement et morphologiquement éloignées serait relativement limité, une fois atteint un niveau minimal en $F_{\mathrm{L} 2}$ et avant l'intervention de compétences spécifiques au français.

\section{a b s t r a c t}

Keywords:

Cross-linguistic transfer

Morphological awareness

Linear/nonlinear morphology

Word reading

Second language learning

There is a great deal of evidence to support the role of morphologica awareness in reading development. However, towards second language acquisition, transfer of morphological awareness from first to second language (L1 to L2) is still discussed. The underlying question concerns the extent to which morphological awareness is a specific or universal process, and its dependence of linguistic features. The aim of this study is to examine cross-linguistic transfer of morphological awareness from Arabic L1 to French L2 (morphological awareness and word reading) at different level of learning French L2 $\left(\mathrm{F}_{\mathrm{L} 2}\right)$. 106 Tunisian children, whose first language is Arabic, in 1 st $(\mathrm{n}=29$; mean age; $8 ; 10$ years $), 2 \mathrm{nd}(\mathrm{n}=33$; mean age; $9 ; 9$ years $)$ or 3 rd year $(n=44 ;$ mean age; $10 ; 10$ years $)$ of learning $F_{\mathrm{L} 2}$ participated to this study. Their morphological awareness (inflectional or derivational oddity detection tasks) and their performance in word reading (one minute test) were assessed in standard Arabic and French, as well as their vocabulary knowledge in French. A series of fixed-order hierarchical regression analysis was performed on derivational awareness performances, inflectional awareness performances and word reading performances in $\mathrm{F}_{\mathrm{L} 2}$, controlling for effects of other important variables (e.g. French vocabulary, French morphological awareness with word reading scores as outcome variable and French inflectional or derivational awareness with French inflectional or derivational awareness scores as outcome variable respectively, Arabic word reading, etc.). Results show significant contributions of L1 morpho-derivational awareness on $\mathrm{F}_{\mathrm{L} 2}$ morpho-derivational awareness in $2 \mathrm{nd}$ year $(12 \%)$, and of $\mathrm{L} 1$ morphological awareness on reading words $\mathrm{F}_{\mathrm{L} 2}$ in $3^{\text {rd }}$ year $(5 \%)$. These results confirm the cross-linguistic transfer of morphological awareness, particularly derivational, from L1 to L2 among alphabetic - but orthographically and morphologically distances - languages. They also suggest that such a transfer could be relatively limited. It could appear during learning process after achieving a threshold in $\mathrm{F}_{\mathrm{L} 2}$ and before specific L2 skills take place. Thus, beyond the morphological opacity of Arabic (nonlinear morphology) and linguistic distance between Arabic and French, the morphological transfer could appear on rich and important morphological dimension in L1 and suggest dealing with the nature of cross-linguistic abilities in depth. 


\section{Introduction}

La notion de transfert interlangue rend compte de l'influence des expériences de la lecture en langue première (L1) sur 1'apprentissage d'une langue seconde (L2) et réciproquement et permet de modéliser les compétences relevant de processus spécifiques dépendants des aspects formels des langues ou de processus centraux indépendants de ceux-ci (Cummins, 2000 ; Koda, 2008).

Le transfert interlangue de la conscience phonologique et de la contribution de cette dernière à la lecture a été largement observé (Bialystok, McBride-Chang, \& Luk, 2005; Kim, 2009). Il confirme le caractère universel de la conscience phonologique postulé par l'hypothèse des processus centraux (Geva \& Siegel, 2000 ; Gleitman, 1985) affirmant que l'acquisition de la lecture, quelle que soit la langue, est sous-tendue par des processus cognitifs identiques. En d'autres termes, il existerait des mécanismes communs aux deux langues, s'appliquant en dépit des variations de surface. La nature exacte de ces processus reste toutefois à préciser, certains auteurs envisageant des mécanismes tels que ceux mobilisant la mémoire verbale de travail ou la dénomination sérielle (Geva \& Siegel, 2000).

En revanche, peu de recherches ont examiné le transfert de la conscience morphologique (CM) et sa contribution à la lecture en L2. Définie comme la connaissance de la structure morphémique des mots et l'habileté à analyser et manipuler cette structure (Carlisle, 1995), son rôle lors de la lecture est bien étayé (e.g. Carlisle, McBride-Chang, Nagy, \& Nunes, 2010 ; Kirby et al., 2012). En effet, sa contribution à la reconnaissance de mots écrits et à la compréhension en lecture a été mis en évidence dans différentes langues (Abu-Rabia, Share, \& Mansour, 2003 ; Casalis \& Louis-Alexandre, 2000 ; Levesque, Kieffer, \& Deacon, 2017 ; Zhang, 2014). Ce constat est valable en L1 comme en L2 (e.g. Ramirez, Chen, Geva, \& Luo, 2011; Kieffer, Biancarosa, \& Mancilla-Martinez, 2013) ce qui pose la question des liens pouvant unir ces habiletés dans deux langues et donc du transfert interlangue de la CM.

\subsection{Structure morphologique du français}

Le français, langue indo-européenne romane, est considéré comme orthographiquement opaque (Ziegler et al., 2010). Certaines inconsistances orthographiques peuvent toutefois être levées par la morphologie. Les morphogrammes permettent de coder à l'écrit les relations morphologiques entre les mots (e.g. gras $\rightarrow$ grasse; acte $\rightarrow$ action). La structure morphologique des mots français est linéaire : les affixes s'ajoutent à la base sans généralement en altérer l'intégrité orthographique. Comme dans d'autres langues, il convient de distinguer ce qui relève :

- de la morphologie flexionnelle dont les modifications syntaxiques permettent d'adapter le mot au contexte de la phrase sans en modifier le sens (e.g. grand/grande);

- de la morphologie dérivationnelle, à fonction lexico-sémantique car à l'origine de la formation de nouveaux mots (e.g. réseautage construit avec réseau et -age).

La morphologie flexionnelle est essentiellement composée de suffixes accolés à une racine (e.g. chant-ent, gris-e-s) alors que la morphologie dérivationnelle permet l'ajout de préfixe et de suffixes (e.g. dé-coller; fleur-iste).

\subsection{Structure morphologique de l'arabe}

En arabe il existe deux formes d'écriture avec ou sans voyelles, l'écriture vocalisée, très transparente, étant généralement employée en début d'apprentissage (Ammar, 2002). Cette caractéristique renvoie à une structure morphologique bidimensionnelle racine consonantique/schème vocalique (Saiegh-Haddad \& Henkin-Roitfarb, 2014). La racine code une signification générale et abstraite (e.g كتب $[\mathrm{ktb}]=$ ce qui se rapporte à l'écrit), squelette consonantique qui n'a pas d'existence lexicale. La formation des mots nécessite donc l'intrication d'un schème à la racine. Ce schème contient des informations sémantiques et syntaxiques et fournit une forme lexicale et grammaticale au mot en complétant le sens générique de la racine. Il s'agit d'un pattern dérivationnel interne principalement composé de voyelles prenant souvent la forme de signes diacritiques absents en écriture non 
vocalisée. Par exemple, كَاتِب ([katib] = celui qui écrit) est formé par l'application du schème nominal [-a-i-] désignant l'agent de l'action, à la racine كتب ([ktb]). L'aspect non linéaire de la morphologie dérivationnelle arabe se traduit ainsi par une rupture de la forme orthographique et phonologique des unités morphologiques. En outre, le lien sémantique entre les mots de la même famille peut

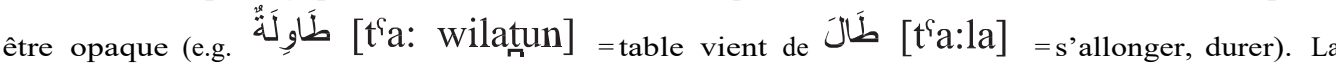
morphologie de l'arabe se caractérise donc par son opacité, liée à la rupture formelle de la racine et à la distance sémantique fréquente entre la racine et le mot construit (Saiegh-Haddad \& Geva, 2008). Cette structure bidimensionnelle concernant une large majorité du lexique, la morphologie de l'arabe se caractérise par sa richesse et sa productivité (Abu-Rabia, 2007). De plus, il existe de nombreuses marques flexionnelles en début ou en fin des mots. Par exemple, pour la conjugaison verbale, les flexions apportent généralement des informations sur le genre et le nombre des trois personnes distinguées de sorte qu'une conjugaison peut comporter jusqu'à 13 formes différentes. De même, les flexions portées par les noms et les adjectifs peuvent être des marques de genre, de nombre, de cas ou de détermination (e.g. féminin construit par l'ajout du ta' marbûta $\ddot{\theta}^{\circ}$ ) à la fin du nom masculin). Comparativement à la morphologie dérivationnelle, la morphologie flexionnelle de l'arabe a une structure plus linéaire. Ainsi, la dichotomie morphologie flexionnelle vs dérivationnelle renvoie généralement au contraste linéaire vs non linéaire des structures morphologiques (Abu-Rabia, 2007).

Bien que très contrastées quant à leur structure, le français et encore plus clairement l'arabe, sont des langues à morphologie riche.

\section{3. $\mathrm{CM}$ et lecture en langues francaise et arabe}

En français, l'impact de la CM flexionnelle (CMflex)et dérivationnelle (CMdériv) sur la lecture a été démontré, dès le début de son apprentissage, s'ajoutant au rôle primordial de la conscience phonologique.

S'intéressant à la CMdériv, Marec-Breton et Gombert (2007) trouvent qu'en $1^{\mathrm{e}}$ année, une tâche de création de néologismes construits morphologiquement (e.g. produire lampette pour compléter la phrase " une petite lampe est une _ ") explique $15 \%$ de la variance en lecture de mots s'ajoutant au rôle toujours plus important de la conscience phonémique. Également, Sanchez, Ecalle, et Magnan (2012) montrent que la CMdériv mesurée en grande section de maternelle (GSM) explique une part indépendante de la variance en lecture de $\operatorname{mot}(5 \%)$ en $1^{\mathrm{e}}$ année et en lecture de mots et de pseudo-mots (4 et $12 \%$ ) en $2^{\mathrm{e}}$ année. Ces résultats corroborent ceux obtenus par Colé, Royer, Lewers, et Casalis (2004) montrant que les enfants ayant de bonnes connaissances morphologiques implicites en $1^{\mathrm{e}}$ année et explicites en $2^{\mathrm{e}}$ et $3^{\mathrm{e}}$ années sont les meilleurs lecteurs. Cependant, en français, peu de recherches se sont intéressées à la CMflex ou conjointement à la CMdériv et CMflex. À notre connaissance, seuls Casalis et Louis-Alexandre (2000), par une étude longitudinale allant de la GSM à la $2^{\mathrm{e}}$ année, montrent que la CMflex puis la CMdériv, bien que n'atteignant pas de scores plafonds interviennent lors de la lecture. Si la conscience phonémique est le meilleur prédicteur de la reconnaissance des mots écrits en $1^{\mathrm{e}}$ année, cette dernière est principalement expliquée, en $2^{\mathrm{e}}$ année, par la CMdériv. Ces résultats sont consistants avec ceux trouvés dans d'autres langues montrant que si la CMdériv se développe moins précocement que la CMflex, elle pourrait constituer un meilleur prédicteur de la réussite en lecture que la CMflex car elle intègre plus de dimensions linguistiques (Kuo \& Anderson, 2006).

En arabe, l'utilisation de la CMdériv pourrait être capitale pour lire, les indices morphologiques permettant d'identifier la structure vocalique des mots, marquée par des signes diacritiques moins saillants que les consonnes. Cependant, certaines voyelles sont également véhiculées par la morphologie flexionnelle, également très présente en arabe, ce qui rend cette dimension également importante. Les recherches menées sur la lecture en arabe chez le lecteur expert ont ainsi souligné l'utilisation des indices morphologiques, en particulier dérivationnels, pour identifier les mots écrits (Boudelaa, 2014) Les travaux qui ont étudié ce phénomène au cours de l'apprentissage de la lecture confirment le rôle de la CM. Parmi les plus récents, l'étude longitudinale de la GSM à la $2^{\mathrm{e}}$ année d'Abu Hammad, Ibrahim, et Share (2014) montre que, même si le meilleur prédicteur de la lecture en arabe vocalisé de $2^{\mathrm{e}}$ année est la conscience phonémique, la CMflex apporte une contribution unique $(9 \%)$ au moins équivalente 
à d'autres mesures infra-lexicales. Boukadida, Gombert, et Maaouia (2009) observent, en $1^{\mathrm{e}}$ année, une contribution similaire de la CMdériv. Ainsi, en dépit de l'opacité morphologique de l'arabe écrit, les compétences morphologiques constituent un facteur déterminant de l'apprentissage de la lecture même vocalisée. Abu-Rabia (2007) a étudié 1'implication de la CM (CMflex et CMdériv) lors de la lecture de mots vocalisés en arabe, chez des élèves normo-lecteurs ou dyslexiques en $3^{\mathrm{e}}, 6^{\mathrm{e}}, 9^{\mathrm{e}}$ et $12^{\mathrm{e}}$ années. Les résultats font apparaître que la CMdériv est, avec les compétences orthographiques, le meilleur prédicteur des habiletés en lecture. Ces résultats confirment que la lecture en arabe s'appuie sur la structure morpho-dérivationnelle des mots écrits. Abu-Rabia (2007) observe aussi que l'application correcte des voyelles à la fin des mots, relevant d'une CMflex, est systématiquement déficitaire chez les enfants dyslexiques mais n'est pas un prédicteur de l'exactitude en lecture de mots. Le traitement des aspects morpho-flexionnels véhiculés par les voyelles brèves pourrait avoir une implication plus limitée sur la lecture que ceux de la morphologie dérivationnelle. Al Ghanem et Kearns (2015) confirment l'augmentation de la contribution de la CM avec l'avancée dans les apprentissages en particulier chez les meilleurs lecteurs, les faibles lecteurs ayant des difficultés à identifier les racines.

\subsection{Relations interlangues et transfert de la $\mathrm{CM}$}

L'étude des mécanismes mettant en relation L1 et L2 fait régulièrement référence à la notion de transfert qui a donné lieu à plusieurs controverses et postulats théoriques. Tardif $\left(1999^{5}\right)$ le définit comme un " mécanisme cognitif qui consiste à utiliser dans une tâche cible, une connaissance construite ou une compétence développée dans une tâche source» (p. 58). Ainsi, le transfert rend compte de la façon dont le développement d'habiletés dans une langue (e.g. la L1) est utilisé et facilite le développement dans une autre (e.g. la L2) (Genesee, Geva, Dressler, \& Kamil, 2008; Kuo \& Anderson, 2010). Empiriquement, le transfert interlangue a été classiquement étudié à l'aide d'analyses corrélationnelles. D'autres méthodologies ont toutefois été utilisées dans l'étude des liens entre L1 et L2 ou de 1'impact des habiletés développées dans une langue sur les habiletés développées dans une autre. Nous tenterons d'en donner un aperçu en présentant les recherches focalisées sur la dimension morphologique.

Dans ce champ de recherche, les relations interlangues ont notamment été étudiées en fonction des similarités vs. disparités entre les langues. Chez des apprenants bilingues espagnol-anglais de $4^{\mathrm{e}}$ et $7^{\mathrm{e}}$ années, Ramirez, Chen, et Pasquarella (2013) montrent qu'il est plus facile d'extraire la signification de mots dérivés en anglais lorsque la base de ces mots sont des cognates (i.e. partagent le même sens, la même orthographe et forme comme " conversion " en anglais et " conversíon " espagnol). La relation entre la CMdériv en espagnol et le vocabulaire en anglais dépend donc de la similarité sémantique et formelle entre les langues. De même, chez des anglophones scolarisés dans un programme d'immersion en français, Hipfner-Boucher, Lam, Chen, et Deacon (2015) montrent que le statut de cognates a un impact positif sur les performances de traduction français-anglais de mots dérivés en $2^{\mathrm{e}}$ et $3^{\mathrm{e}}$ années.

Afin d'étudier le transfert de la CM, Deacon, Wade-Woolley, et Kirby (2007) ont examiné la contribution de la CMflex au développement de la lecture de la $1^{\mathrm{e}}$ à la $3^{\mathrm{e}}$ année chez des enfants apprenant à lire en anglais L1 et en français L2 $\left(\mathrm{F}_{\mathrm{L} 2}\right)$. Leurs résultats montrent que la CMflex en L1 mesurée en $1^{\mathrm{e}}$ année est corrélée avec la $\mathrm{CMflex}$ en $\mathrm{F}_{\mathrm{L} 2}$ à tous les niveaux scolaires et contribue à la lecture de mots en L2 (6 à 17 \% de variance expliquée). De même, Ramirez, Chen, Geva, et Kiefer (2010) montrent, chez des apprenants hispanophones de $4^{\mathrm{e}}$ et $7^{\mathrm{e}}$ année vivant au Canada anglophone, que la CMdériv en espagnol est corrélée à la CMdériv en anglais L2 $\left(\mathrm{E}_{\mathrm{L} 2}\right)$ et contribue à la lecture de mots en L2 $(5 \%$ de variance expliquée). Auprès de la même population, Ramirez et al. (2013) observent également la contribution de la CMderiv L1 à la CMdériv et à la lecture de mots L2. Chez des enfants scolarisés en $2^{\mathrm{e}}, 3^{\mathrm{e}}$ et $4^{\mathrm{e}}$ année aux États-Unis et suivant un apprentissage bilingue coréen-anglais, Wang, Ko, et Choi (2009) observent qu'en dépit des différences orthographiques entre les langues la CMdériv en anglais prédit le niveau de lecture en coréen et la CMdériv en coréen prédit le niveau de lecture en anglais ( 7 et $4 \%$ de variance expliquée), ceci parce que l'anglais et le coréen partagent des structures morphologiques

\footnotetext{
5 Cité par Legendre (2001)
} 
similaires (e.g. même processus de suffixation en coréen: 과학 $\rightarrow$ 과학자 et en anglais : science $\rightarrow$ scientist). Enfin, Zhang, Koda, et Leong (2016) auprès d'enfants de $3^{\mathrm{e}}$ année, de langue maternelle malais mais de langue dominante anglais suivis pendant six mois, mettent en évidence un transfert de la CMderiv en anglais sur l'habileté à effectuer des inférences lexicales permettant d'identifier la signification d'un mot dérivé en malais.

Lorsque les langues ont des structures et règles morphologiques plus éloignées, les résultats sont plus disparates. Dans une étude auprès de bilingues chinois-anglais scolarisés de la $1^{\mathrm{e}}$ à la $4^{\mathrm{e}}$ année, Wang, Cheng, et Chen (2006) n'observent pas de contribution de la CM (dérivationnelle et compositionnelle) en chinois L1 à la lecture en $\mathrm{E}_{\mathrm{L} 2}$. Saiegh-Haddad et Geva (2008) auprès d'élèves anglophones scolarisés dans une école bilingue anglais-arabe de la $3^{\mathrm{e}}$ à la $6^{\mathrm{e}}$ année n'observent pas non plus de corrélations significatives entre la CMdériv dans ces deux langues. En outre, la contribution de la CM en anglais L1 à la lecture en arabe L2 n'est pas significative mais la contribution interlangue inverse l'est. L'opacité orthographique de l'anglais rendrait nécessaire la coordination des dimensions phonologique, orthographique et lexicale lors de la lecture, comme c'est le cas pour analyser la structure morphologique en arabe, particulièrement opaque.

Pourtant, au moins deux études sur l'hébreu et l'anglais apportent des données favorables au transfert morphologique entre langues sémitiques et indo-européennes. Chez des élèves de L1 hébreu en $2^{\mathrm{e}}$ année d'apprentissage de l'E $E_{\mathrm{L} 2}$, Kahn-Horwitz, Shimron, et Spark (2005), ont montré le rôle prédicteur de la CMdériv en hébreu L1 sur la lecture de pseudo-mots et la compréhension en lecture en $\mathrm{E}_{\mathrm{L} 2}$. Ainsi, malgré les différences de surface entre L1 et L2, les apprenants peuvent transférer leur CM. Schiff et Calif (2007) ont, pour leur part, montré qu'en $4^{\mathrm{e}}$ année d'apprentissage de $1^{\prime} \mathrm{E}_{\mathrm{L} 2}$, la lecture de mots dans cette langue est expliquée par la combinaison de la conscience orthographico-phonologique et de la CMdériv en hébreu L1 et en L2. En d'autres termes, si le transfert est bien effectifla CM en L1 doit être couplée avec des connaissances en L2 pour jouer un rôle sur la lecture en $E_{\mathrm{L} 2}$.

Dans ces derniers travaux, les auteurs envisagent le lien interlangue et le transfert au travers d'études corrélationnelles. Peu s'intéressent aux autres effets que ceux sur les performances en lecture. Ainsi, la contribution de la CM en L1 au développement de la CM en L2 reste peu étudiée. À notre connaissance, seuls Schiff et Calif (2007) font apparaître, en $4^{\mathrm{e}}$ année d'apprentissage de l'E $\mathrm{E}_{\mathrm{L} 2}$, que la CMdériv en hébreu L1 prédit la CMdériv en $\mathrm{E}_{\mathrm{L} 2}$ (11 \% de variance expliquée). Les compétences morphologiques mesurées par les auteurs en hébreu portaient sur des structures morphologiques linéaires comme c'est le cas en anglais. Cette similarité morphologique serait ainsi à l'origine de la relation entre les deux langues. Pourtant, l'hébreu utilise principalement une morphologie non linéaire ce qui rend le matériel linguistique employé par Schiff et Calif (2007) peu représentatif de l'ensemble des constructions morphologiques de cette langue. À ce sujet, les travaux menées sur des langues dont les systèmes d'écriture diffèrent fortement (e.g. chinois et anglais) insistent sur 1'impact de la distance linguistique entre L1 et L2 sur le transfert morphologique (Ke \& Xiao, 2015 ; Zhang, 2013).

L'ensemble de ces résultats, s'ils ne remettent pas en cause la définition du transfert évoqué précédemment, souligne la nécessité d'identifier des facteurs modérateurs dans la mise en place du transfert. Plusieurs auteurs insistent sur le fait que l'observation du transfert de la CM est dépendant du degré de proximité structurale des langues (Wang et al., 2009). D'autres études mettent en avant d'autres facteurs, comme la transparence morphologique (Saiegh-Haddad \& Geva, 2008). Il se pourrait donc que certaines caractéristiques linguistiques des langues impactent le transfert, ce qui signifierait que les processus centraux universaux doivent être complétés par la prise en compte de processus spécifiques aux langues et aux systèmes d'écriture.

\subsection{Objectifs et hypothèses}

Cette étude a pour objectif d'étudier le transfert interlangue de la $\mathrm{CM}$ en arabe $\mathrm{L} 1\left(\mathrm{~A}_{\mathrm{L} 1}\right)$ vers la $\mathrm{CM}$ et la lecture en $\mathrm{F}_{\mathrm{L} 2}$. Étant donné l'importance de la $\mathrm{CM}$ dans ces deux langues, les liens entre ces connaissances méritent d'être questionnés. En effet, le transfert morphologique n'a pas été suffisamment étudié pour en affirmer le caractère universel ou spécifique. L'étude d'une L1 sémitique comme 1'arabe et d'une L2 indo-européenne comme le français s'avère particulièrement intéressante car ces deux langues sont très contrastées en termes de caractéristiques morphologiques et de transparence 
orthographique tout en étant toutes deux alphabétiques. Une telle étude permettrait d'identifier si la distance entres les constructions morphologiques entrave ou non le transfert de la CM d'une L1 particulièrement riche morphologiquement à une L2 pour laquelle la morphologie est également une dimension importante à l'écrit (Casalis, Quémart, \& Duncan, 2015). Parallèlement à 1'impact de la distance structurale des langues, un intérêt particulier sera ainsi accordé ici à la richesse et à la prégnance de la morphologie dans les systèmes d'écriture.

Nous nous intéresserons aux relations entre la CM (CMflex et CMdériv) développée en $\mathrm{A}_{\mathrm{L} 1}$ et en $\mathrm{F}_{\mathrm{L} 2}$ au cours des apprentissages. En lien avec le postulat de l'intervention de processus centraux, nous formulons l'hypothèse d'un transfert de la L1 sur la L2 qui devrait s'observer à tous ou partie des niveaux étudiés, tant pour la CMdériv que la CMflex. L'hypothèse alternative de l'intervention de processus spécifiques serait en revanche confortée par l'observation d'un transfert interlangue limité à la CM reposant sur une morphologie linéaire (i.e. la morphologie flexionnelle). Dans un tel cas, lorsque les constructions reposent dans une langue sur une morphologie non linéaire et dans l'autre sur une morphologie linéaire, la non-similarité des constructions se traduirait par une absence de transfert.

Ensuite, nous chercherons à rendre compte du transfert de la $\mathrm{CM}$ (CMflex et CMdériv) en $\mathrm{A}_{\mathrm{L} 1}$ sur la lecture de mots en $\mathrm{F}_{\mathrm{L} 2}$. Les études suggérant un tel transfert d'une L1 sémitique sur la lecture en L2 n'ayant jusqu'alors jamais porté sur $1 \mathrm{la} 1^{\mathrm{e}}$ année d'apprentissage, nous tenterons d'identifier à partir de quand il est opérant. Il s'agira enfin de vérifier si les aspects morphologiques dérivationnels, qui sont les plus déterminants pour lire en arabe, sont bien ceux les plus impliqués lors de la lecture en L2 et cela à différents niveaux scolaires.

Avant de tester ces hypothèses, nous vérifierons dans un premier temps le développement des habiletés morphologiques dans les deux langues (Berninger, Abott, Nagy, \& Carlisle, 2010) en testant $1^{\prime}$ 'effet du niveau scolaire ( $3^{\mathrm{e}}, 4^{\mathrm{e}}$ et $5^{\mathrm{e}}$ années ou $1^{\mathrm{e}}$ année, $2^{\mathrm{e}}$ année et $3^{\mathrm{e}}$ année en $\mathrm{F}_{\mathrm{L} 2}$ ). Cet objectif, bien que secondaire, constitue un préalable dans la mesure où les hypothèses sur le transfert reposent sur 1'émergence des CM en L1 et en L2.

\section{Méthode}

\subsection{Participants}

Cent six élèves (54 garçons et 52 filles) tunisiens scolarisés en $3^{\mathrm{e}}, 4^{\mathrm{e}}$ et $5^{\mathrm{e}}$ années dans des établissements primaires publics de Tunis ont participé à cette étude. Tous sont de langue maternelle arabe, n'ont ni avance ni retard scolaire et ont suivi un enseignement du français depuis la $3^{\mathrm{e}}$ année. Les élèves de $3^{\mathrm{e}}$ année de scolarité $(\mathrm{n}=29$, âge moyen $=8 ; 10$ ans, $\mathrm{a}=3,38$ mois $)$ sont donc en $1^{\mathrm{e}}$ année de français, les élèves de $4^{\mathrm{e}}$ année ( $\mathrm{n}=33$, âge moyen $=9 ; 9$ ans, $\mathrm{a}=3,06$ mois) sont en $2^{\mathrm{e}}$ année de français et ceux de $5^{\mathrm{e}}$ année $(\mathrm{n}=44$, âge moyen $=10 ; 10$ ans, $\mathrm{a}=3,06$ mois $)$ sont en $3^{\mathrm{e}}$ année de français.

\subsection{Matériel}

Des tâches isomorphes en $\mathrm{F}_{\mathrm{L} 2}$ et $\mathrm{A}_{\mathrm{L} 1}$ ont été administrées.

\subsection{Tâches en $\mathrm{F}_{\mathrm{L} 2}$}

Tâche chronométrée de lecture de mots : les compétences d'identification de mots écrits en français sont évaluées à l'aide du test LUM (Khomsi, 1999). Il composée de 108 mots de différentes catégories grammaticales et de différentes longueurs, variant en termes de fréquence, de régularité et de complexité, de $4 \%$ de formes fléchies et de $19 \%$ de formes dérivées.

Tâches de CM : Tâche de détection d'intrus morpho-flexionnel (cf. Annexe 1) : Cette épreuve évalue les compétences à identifier consciemment les aspects flexionnels des mots écrits. Les participants doivent déterminer, parmi deux propositions et malgré les apparences phonographiques, le nom non assigné d'une marque de genre, ou le verbe non conjugué comme le mot cible (6 items sur 12 concernent le féminin de noms ou d'adjectifs et 6 les désinences du présent de l'indicatif). Les aspects sémantiques, la structure phonologique et graphique, la fréquence (cf. MANULEX ; Lété, 
Sprenger-Charolles, \& Colé, 2004) et la catégorie grammaticale des mots ont été contrôlés ${ }^{6}$. Le coefficient de cohérence interne est faible $\left(c_{c}=0,39\right)$.

Tâche de détection d'intrus morpho-dérivationnel : Cette épreuve évalue les compétences permettant d'identifier la construction de mots dérivés. Les participants doivent déterminer parmi deux mots proposés celui qui, malgré les apparences phonographiques, ne fait pas apparaître la même construction morphologique qu'un mot cible. 6 items sur 12 concernent l'ajout de suffixes (-ier, -eur) et 6 l'ajout de préfixes (dé-, re-). Le coefficient de cohérence interne s'approche d'une valeur acceptable $(c=0,59)$.

Test de vocabulaire : Les connaissances lexicales en Francais L2 sont évaluées afin de s'assurer que le niveau de vocabulaire est suffisant pour résoudre des tâches impliquant des mots français. Elles sont évaluées à l'aide du " test de vocabulaire passif " (Deltour \& Hupkens, 1980) adapté pour une passation collective. Les participants doivent choisir parmi 6 images, celle correspondant au mot présenté oralement ( 30 items). Un point est attribué à chaque bonne réponse et $1 / 2$ point à une image sémantiquement proche. Le coefficient de cohérence interne est satisfaisant $\left(c_{c}=0,93\right)$.

\subsection{Tâches en $A_{L 1}$}

Épreuve chronométrée de lecture de mots : les compétences d'identification de mots écrits en arabe standard vocalisé sont évaluées à l'aide d'une épreuve chronométrée de lecture de mots isolés, adaptation en arabe du test Lecture en Une Minute (LUM) de Khomsi (1999) (Khomsi et l'équipe de l'ISSHT, travaux non publiés). Une liste de 108 mots doit être lue à voix haute le plus rapidement possible. Elle se compose de près de $70 \%$ de mots dont on retrouve la structure racine/schème, de $78 \%$ de substantifs

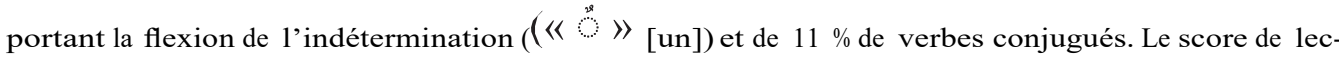
ture correspond au nombre de mots lus correctement en une minute. Cette mesure permet de savoir si les apprenants maîtrisent la lecture dans leur L1. Bien que l'arabe non vocalisé soit introduit aux niveaux scolaires étudiés, une version vocalisée a été choisie car la vocalisation est particulièrement nécessaire lorsqu'il s'agit de lire des mots en dehors de tout contexte.

Tâches de CM (cf. Annexe 1) : Les phénomènes morphologiques choisis ont été sélectionnés de facon à être connus des élèves et à permettre l'utilisation de formes pseudo-dérivées ou pseudo-fléchies. Compte tenu de l'ampleur des formes fléchies et dérivées en arabe, nous avons également souhaité proposer dans ces tâches une diversité de formes, nominale ou verbale, avec suffixes, préfixes ou schème interne.

Épreuve de détection d'intrus morpho-flexionnel : Cette épreuve évalue les compétences permettant d'identifier consciemment les marques flexionnelles de mots écrits. Les participants doivent déterminer, parmi deux propositions et malgré les apparences phonographiques, le verbe non marqué par la conjugaison à la première personne du singulier de l'inaccompli (préfixe $\{$; 6 items sur 12) exprimant un procès en cours et non achevé ou le nom non assigné d'une marque de genre (suffixe $\ddot{0} ; 6$ items). Ces marques flexionnelles ont donc la caractéristique d'utiliser des affixes, ce qui les rapprochent de la structure morphologique du francais. Les aspects sémantiques, la structure phonologique et graphique $^{7}$, la familiarité et le rapport avec le lexique en arabe dialectal des mots ${ }^{8}$ ont été contrôlés. Le coefficient de cohérence interne s'approche d'une valeur acceptable $(c=0,61)$.

Épreuve de détection d'intrus morpho-dérivationnel : Cette épreuve évalue les compétences permettant d'identifier la construction morphologique de mots dérivés écrits. Sur le même principe que la tâche précédente, les participants doivent, parmi deux mots proposés, déterminer celui qui, malgré les apparences phonographiques, ne fait pas apparaître la dichotomie racine/schème présent dans

\footnotetext{
${ }^{6}$ Il en est de même pour la tâche morpho-dérivationnelle.

7 Entre l'intrus et la bonne réponse, aucun des deux ne se rapproche sémantiquement, phonologiquement ou formellement plus du mot cible, ce contrôle étant effectué sur toutes les tâches morphologiques.

${ }^{8}$ Un pré-test a permis de mesurer la familiarité de chaque mot et de faire en sorte que les deux propositions de réponse soient équivalentes sur ce point. De plus l'équivalent en arabe dialectal des mots est proche. Ces deux aspects de contrôle ont aussi été appliqués lors de la construction de la tâche morpho-dérivationnelle en arabe.
} 
Tableau 1

Moyennes (écarts-types) obtenues aux tâches $\mathrm{A}_{\mathrm{L} 1}$ et $\mathrm{F}_{\mathrm{L} 2}$ aux trois niveaux.

\begin{tabular}{lccc}
\hline Tâches & $\begin{array}{l}1^{\mathrm{e}} \text { année } \mathrm{F}_{\mathrm{L} 2} \\
(\mathrm{n}=29)\end{array}$ & $\begin{array}{l}2^{\mathrm{e}} \text { année } \mathrm{F}_{\mathrm{L} 2} \\
(\mathrm{n}=33)\end{array}$ & $\begin{array}{l}3^{\mathrm{e}} \text { année } \mathrm{F}_{\mathrm{L} 2} \\
(\mathrm{n}=44)\end{array}$ \\
\hline En $\mathrm{AL}_{\mathrm{L} 1}$ & & & $74,59(18,67)$ \\
$\quad$ Lecture mots & $57,17(19,37)$ & $65,21(15,41)$ & $9,11(2,22)$ \\
CMflex & $7,38(2,38)$ & $7,21(1,95)$ & $9,34(2,05)$ \\
CMdériv & $8,66(1,67)$ & $8,52(1,64)$ & $45,64(16,92)$ \\
En F & & & $23,05(4,06)$ \\
Lecture mots & $17,00(8,56)$ & $35,12(11,90)$ & $8,41(2,14)$ \\
Vocabulaire (/30) & $15,3(3,80)$ & $19,38(2,70)$ & $9,18(2,05)$ \\
CMflex & $7,07(1,71)$ & $7,21(1,45)$ & $8,42(1,73)$ \\
CMdériv & $7,07(1,51)$ & & \\
\hline
\end{tabular}

un mot cible. 6 items sur 12 concernent la formation de substantifs $^{9}$ (et 6 la formation de verbes ${ }^{10}$ ). Ces formes dérivées ont la caractéristique d'utiliser des schèmes internes, ce qui les éloignent de la structure morphologique linéaire du français. Le coefficient de cohérence interne s'approche ici aussi d'une valeur acceptable $\left(c_{c}=0,62\right)$

\subsection{Procédure}

Les passations ont eu lieu 20 semaines après le début de l'année scolaire. Les tâches morphologiques (L1 et L2) ont été présentées en passation collective, oralement avec un support écrit pour ne pas saturer la mémoire de travail par les traitements liés au décodage. Les participants devaient entourer la réponse choisie. Elles étaient présentées d'abord en arabe puis en français afin que les consignes soient présentées en premier dans la langue la mieux maîtrisée. L'ordre de présentation des tâches flexionnelles et dérivationnelles était contrebalancé de façon que la résolution de l'une n'impacte pas la résolution de l'autre. Le test de vocabulaire a été administré en passation collective, les tâches de lecture se faisant en passation individuelle avec contre-balancement de l'ordre des langues.

\section{Résultats}

Des analyses de variance ont été appliquées sur les résultats obtenus aux différentes tâches puis des analyses de régressions hiérarchiques destinées à tester les hypothèses. Il a été vérifié que les coefficients de symétrie et d'aplatissement se rapprochaient de ceux de la loi normale (compris entre $-0,36$ et 0,22 ). Les scores aux différentes tâches dans les deux langues et pour les trois niveaux sont présentés dans le Tableau 1. Pour les tâches de CM, il faut souligner que la faiblesse des \& de Cronbach signale un risque d'erreur de mesure.

Pour les tâches de CM, le paradigme de détection d'intrus proposant deux choix possibles de réponse, il a d'abord été vérifié que les performances se différencient des réponses produites de façon aléatoire (Howel, 1998 ; voir aussi Hipfner-Boucher et al., 2015). Le critère retenu pour établir cette distinction est la valeur 7,7 c'est-à-dire située à un écart-type $(1,7)$ de la réponse attendue par le hasard (6/12). Les tests $\mathrm{t}$ de comparaison à la valeur 7,7 montrent qu'en $1^{\mathrm{e}}$ année, seuls les scores à la tâche morpho-dérivationnelle en $A_{L 1}$ sont significativement supérieurs à cette valeur $(t(28)=3,07, p<0,01)$. En $2^{\mathrm{e}}$ année, les scores aux tâches de CMdériv dans les deux langues s'en distinguent $(\mathrm{t}(32)=2,85$, $\mathrm{p}<0,01$ et $\mathrm{t}(32)=2,85, \mathrm{p}<0,01$ respectivement en arabe et en français) et en $3^{\mathrm{e}}$ année, l'ensemble des

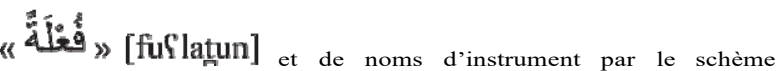

9 Formation de substantifs par le schème

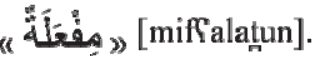

${ }_{10}$ Forme III intensive « Cَ
} 
résultats aux tâches morphologiques (CMdériv et CMflex) dans les deux langues s'en distingue (CMdériv arabe : $\mathrm{t}(43)=5,32, \mathrm{p}<0,001$; CMflex arabe : $\mathrm{t}(43)=4,90, \mathrm{p}<0,001 ;$ CMdériv français : $\mathrm{t}(43)=4,80$, $\mathrm{p}<0,0001$; CMflex français : $\mathrm{t}(43)=2,20, \mathrm{p}<0,05)$.

Ces résultats impliquent que les scores à certaines tâches morphologiques ne soient pas retenus lors des analyses suivantes. Les comparaisons intergroupes n'ont donc pu être menées sur les scores des trois niveaux que pour la tâche CMdériv en $A_{L 1}$, et que sur les scores des élèves de $2^{\mathrm{e}}$ et $3^{\mathrm{e}}$ années à la tâche CMdériven $F_{\mathrm{L} 2}$. Ces analyses ne mettent pas en évidence d'effet du niveau $[\mathrm{F}(2,103)=2,27$; ns et $\mathrm{t}(75)=-1,75 ; \mathrm{ns}]$. Enfin, les comparaisons qui ont été menées, en $3^{\mathrm{e}}$ année, entre les scores aux tâches CMdériv et CMflex en $\mathrm{F}_{\mathrm{L} 2}$ et en $\mathrm{A}_{\mathrm{L} 1}$ ne mettent pas en évidence de différences significatives $[\mathrm{t}(86)=1,7 ; \mathrm{ns}$ et $\mathrm{t}(86)=0,15 ; \mathrm{ns}]$. Ces résultats ne permettent pas de mettre en évidence les progrès en $\mathrm{CM}$ ni en $\mathrm{A}_{\mathrm{L} 1}$ ni en $\mathrm{F}_{\mathrm{L} 2}$, mais les comparaisons avec le hasard laissent entrevoir une meilleure maîtrise des règles morpho-dérivationnelles en arabe dès le $1^{\mathrm{e}}$ niveau étudié et leur émergence en français à partir de la $2^{\mathrm{e}}$ année.

Pour tester l'effet du niveau scolaire sur les scores en vocabulaire $\mathrm{F}_{\mathrm{L} 2}$ et en lecture dans les deux langues, trois analyses de variance ont été menées. Sur les scores en $\mathrm{F}_{\mathrm{L} 2}$ (vocabulaire et lecture), l'effet du niveau est systématiquement significatif $[\mathrm{F}(2,103)=39,97, \mathrm{p}<0,001$ et $\mathrm{F}(2,103)=39,12 ; \mathrm{p}<0,001]$, les résultats des élèves de $3^{\mathrm{e}}$ année $\mathrm{F}_{\mathrm{L} 2}$ étant supérieurs à ceux des élèves de $2^{\mathrm{e}}$ année eux-mêmes supérieurs à ceux de $1^{\mathrm{e}}$ année $\left(\mathrm{p}_{\mathrm{s}}<0,001\right)$. Les scores en vocabulaire avoisinent les $50 \%$ de bonnes réponses en $1^{\mathrm{e}}$ année et dépassent les $60 \%$ dès la $2^{\mathrm{e}}$ année. De même, les scores en lecture augmentent régulièrement. L'effet du niveau scolaire est aussi attesté sur les scores en lecture de mots $\mathrm{A}_{\mathrm{L} 1}[\mathrm{~F}(2$, 103 $=8,47 ; \mathrm{p}<0,001]$, les élèves de $3^{\mathrm{e}}$ année $\mathrm{F}_{\mathrm{L} 2}$ lisant plus de mots arabes que les élèves de $1^{\mathrm{e}}$ et de $2^{\mathrm{e}}$ année $(\mathrm{p}<.001$ et $\mathrm{p}<0,05)$ dont les scores ne se distinguent pas significativement. Ces résultats soulignent les progrès avec l'avancée dans les apprentissages, progrès d'autant plus soutenus en français puisque l'écart en lecture entre la $1^{\mathrm{e}}$ et la $2^{\mathrm{e}}$ années est significatif en français mais pas en arabe. Malgré des écarts importants entre les participants (écarts-types entre 15,41 et 19,37), les scores en lecture de mots en arabe sont d'ailleurs élevés dès la $1^{\mathrm{e}}$ année.

\subsection{Analyses de corrélations}

Avant de tester la contribution de la $\mathrm{CM} \mathrm{A}_{\mathrm{L} 1}$ à la $\mathrm{CM}$ et à la lecture $\mathrm{F}_{\mathrm{L} 2}$, il importe de vérifier l'existence de corrélations entre l'arabe et le français.

\subsection{Corrélations interlangues}

En fonction des scores aux tâches morphologiques se distinguant des réponses au hasard, les performances varient d'un niveau à l'autre. Des analyses de corrélation séparées ont donc été menées en $1^{\mathrm{e}}, 2^{\mathrm{e}}$ et $3^{\mathrm{e}}$ années $\mathrm{F}_{\mathrm{L} 2}$ (cf. Tableau 2).

Tableau 2

Corrélations interlangues entre les scores en lecture de mots et aux tâches de CM à l'intérieur de chaque niveau.

\begin{tabular}{|c|c|c|c|c|}
\hline \multirow[b]{2}{*}{ Tâches $A_{L 1}$} & \multicolumn{4}{|l|}{ Tâches $\mathrm{F}_{\mathrm{L} 2}$} \\
\hline & Lecture de mots & Mdériv & Mflex & Vocabulaire \\
\hline \multicolumn{5}{|c|}{$1^{\mathrm{e}}$ année $\mathrm{F}_{\mathrm{L} 2}(\mathrm{n}=29)$} \\
\hline Lecture mots & $0,64^{* * *}$ & $\mathrm{Nd}$ & $\mathrm{Nd}$ & 0,08 \\
\hline Mdériv & 0,003 & $\mathrm{Nd}$ & $\mathrm{Nd}$ & 0,20 \\
\hline \multicolumn{5}{|c|}{$2^{\mathrm{e}}$ année $\mathrm{F}_{\mathrm{L} 2}(\mathrm{n}=33)$} \\
\hline Lecture mots & $0,55^{* * *}$ & 0,00 & $\mathrm{Nd}$ & 0,34 \\
\hline Mdériv & $0,51^{* *}$ & $0,47^{* *}$ & $\mathrm{Nd}$ & 0,17 \\
\hline \multicolumn{5}{|c|}{$3^{\mathrm{e}}$ année $\mathrm{F}_{\mathrm{L} 2}(\mathrm{n}=44)$} \\
\hline Lecture mots & $0,55^{* * *}$ & $0,45^{* *}$ & 0,26 & 0,02 \\
\hline Mdériv & $0,54^{* * *}$ & $0,43^{* *}$ & $0,31^{*}$ & 0,21 \\
\hline Mflex & 0,11 & 0,22 & 0,20 & 0,07 \\
\hline
\end{tabular}

$\mathrm{Nd}$ : données non disponibles. ${ }^{* * *} \mathrm{r}$ significatifà $\mathrm{p}<0,001,{ }^{* *} \mathrm{p}<0,01,{ }^{*} \mathrm{p}<0,05$. 
Tableau 3

Corrélations intralangues entre les scores en lecture de mots, en vocabulaire et aux tâches de CM F $\mathrm{L}_{2}$ à l'intérieur de chaque niveau.

\begin{tabular}{llll}
\hline Tâches en $\mathrm{F}_{\mathrm{L} 2}$ & Lecture de mots & Mdériv & Mflex \\
\hline $1^{\mathrm{e}}$ année $\mathrm{F}_{\mathrm{L} 2}(\mathrm{n}=29)$ & 0,01 & $\mathrm{Nd}$ & $\mathrm{Nd}$ \\
$\quad$ Vocabulaire & & & $\mathrm{Nd}$ \\
$2^{\mathrm{e}}$ année $\mathrm{F}_{\mathrm{L} 2}(\mathrm{n}=33)$ & $0,38^{*}$ & 0,03 & $\mathrm{Nd}$ \\
$\quad$ Vocabulaire & - & $0,34^{* * * *}$ & \\
Lecture mots & & & $0,33^{*}$ \\
$3^{\mathrm{e}}$ année $\mathrm{F}_{\mathrm{L} 2}(\mathrm{n}=44)$ & $0,51^{* * *}$ & 0,28 & $0,43^{* *}$ \\
Vocabulaire & - & $0,53^{* * *}$ & $0,50^{* *}$ \\
Lecture mots & - & - & \\
Mdériv &
\end{tabular}

$\mathrm{Nd}$ : données non disponibles. ${ }^{* * *} \mathrm{r}$ significatif à $\mathrm{p}<0,001,{ }^{* *} \mathrm{p}<0,01,{ }^{*} \mathrm{p}<0,05,{ }^{* * * *} \mathrm{p}=0,05$.

La corrélation entre les scores CMdériv $\mathrm{A}_{\mathrm{L} 1}$ et la lecture $\mathrm{F}_{\mathrm{L} 2}$ n'est significative qu'à partir de la $2^{\mathrm{e}}$ année $\left(\mathrm{r}_{\mathrm{S}}=0,51\right.$ et 0,54$)$. En $2^{\mathrm{e}}$ et $3^{\mathrm{e}}$ années, niveaux où les scores à la tâche CMdériv $\mathrm{F}_{\mathrm{L} 2}$ sont analysables, ceux-ci sont significativement corrélés à leur homologue en $A_{L 1}\left(r_{S}=0,47\right.$ et 0,43$)$ mais ils ne sont significativement corrélés à la lecture de mots $A_{L 1}$ qu'en $3^{e}$ année $(r=0,45)$. À ce niveau, les performances CMflex $\mathrm{A}_{\mathrm{L} 1}$ ne sont significativement corrélées à aucune autre mesure en $\mathrm{F}_{\mathrm{L} 2}$. À tous les niveaux, une corrélation significative entre les scores en lecture dans les deux langues est observée $\left(\mathrm{r}_{\mathrm{s}}=0,55\right.$ à 0,64$)$.

\subsection{Corrélations intralangue en $\mathrm{F}_{\mathrm{L} 2}$}

L'analyse des corrélations entre les différentes mesures disponibles en $F_{L 2}$ (cf. Tableau 3) montre que la corrélation entre la lecture et le vocabulaire n'est significative qu'en $2^{\mathrm{e}}$ et $3^{\mathrm{e}}$ années $\left(\mathrm{r}_{\mathrm{s}}=0,38\right.$ et 0,51). L'analyse des liens entre CMdériv et les autres variables, examinés à partir de la $2^{\mathrm{e}}$ année, fait apparaître une corrélation entre CMdériv et la lecture de mot en $2^{\mathrm{e}}$ année $(\mathrm{r}=0,34)$ et $3^{\mathrm{e}}$ année $(\mathrm{r}=0,53)$. En revanche la CMdériv n'est jamais corrélée au vocabulaire. En $3^{\mathrm{e}}$ année, l'introduction de la tâche CMflex montre que ces scores sont corrélés aux trois autres mesures en $\mathrm{F}_{\mathrm{L} 2}$ (lecture, vocabulaire et CMdériv; $\mathrm{r}_{\mathrm{s}}=0,33$ à 0,50$)$.

\subsection{Analyses de régression hiérarchiques}

\subsubsection{Régressions hiérarchiques sur la $\mathrm{CMF}_{\mathrm{L} 2}$}

Afin de déterminer la contribution de la $\mathrm{CM}$ mesurée en $\mathrm{A}_{\mathrm{L} 1}$ au développement de son homologue en $\mathrm{F}_{\mathrm{L} 2}$ et en fonction des coefficients de corrélations recueillis précédemment, une série de régressions hiérarchiques a été menée séparément en $2^{\mathrm{e}}$ et $3^{\mathrm{e}}$ années (cf. Tableau 4). En $1^{\mathrm{e}}$ année, la tâche de détection ne permet pas de mettre en évidence une $\mathrm{CM}_{\text {en }} \mathrm{F}_{\mathrm{L} 2}$, ce qui annule toute possibilité d'analyser les facteurs y contribuant. En outre, les mêmes variables n'ayant pas pu être retenues en $2^{\mathrm{e}}$ et $3^{\mathrm{e}}$ années, des procédures distinctes ont été adoptées.

En $2^{\mathrm{e}}$ année, une analyse de régression a été menée avec comme variable dépendante les scores CMdériv $\mathrm{F}_{\mathrm{L} 2}$. En pas 1, la lecture de mots $\mathrm{F}_{\mathrm{L} 2}$ a été introduite ${ }^{11}$ puis les performances CMdériv $\mathrm{A}_{\mathrm{L} 1}$ En $3^{\mathrm{e}}$ année, une première analyse de régression hiérarchique a porté sur les scores CMdériv et une seconde sur les scores CMflex. Dans chacune d'elle, la variable entrée en pas 1 est la lecture $\mathrm{F}_{\mathrm{L} 2}$ L'analyse portant sur la CMdériv $\mathrm{F}_{\mathrm{L} 2}$ comprend en pas 2, la $\mathrm{CMflex}_{\mathrm{F}_{\mathrm{L} 2}}{ }^{12}$ puis en pas 3, la CMdériv $\mathrm{A}_{\mathrm{L} 1}$. Le vocabulaire n'étant corrélé qu'avec les scores CMflex $\mathrm{F}_{\mathrm{L} 2}, 1$ 'analyse de cette dernière variable

\footnotetext{
11 La corrélation entre le $\mathrm{CMdériv}_{\mathrm{L} 2}$ et la lecture $\mathrm{F}_{\mathrm{L} 2}$ ainsi que les caractéristiques des tâches morphologiques et certains éléments théoriques justifient que la lecture soit forcée en pas 1 pour en contrôler les effets (e.g. Carlisle, 2003).

12 Les relations linguistiques mais aussi la corrélation en $3^{\mathrm{e}}$ année entre les performances Mdériv et Mflex $\mathrm{F}_{\mathrm{L} 2}(\mathrm{r}=.50 ; \mathrm{p}<001)$ justifient de contrôler leur effet.
} 
Tableau 4

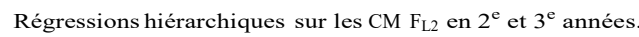

\begin{tabular}{|c|c|c|c|}
\hline & $\checkmark$ & $\mathrm{R}^{2}$ & $\Leftrightarrow \mathrm{R}^{2}$ \\
\hline \multicolumn{4}{|c|}{ Prédictives de la CMdériv $\mathrm{F}_{\mathrm{L} 2} 2^{\mathrm{e}}$ année $(\mathrm{n}=33)$} \\
\hline Pas 1 : lecture mots $\mathrm{F}_{\mathrm{L} 2}$ & 0,14 & 12 & $0,12^{*}$ \\
\hline Pas 2 : CMdériv $A_{L 1}$ & $0,40^{*}$ & 24 & $0,12^{*}$ \\
\hline \multicolumn{4}{|c|}{ Prédictives de la CMdériv $F_{\mathrm{L} 2} 3^{\mathrm{e}}$ année $(\mathrm{n}=44)$} \\
\hline Pas 1 : lecture mots $\mathrm{F}_{\mathrm{L} 2}$ & $0,30^{* * * * *}$ & 0,28 & $28^{* * *}$ \\
\hline Pas 2: CMflex $F_{\mathrm{L} 2}$ & $0,33^{*}$ & 0,37 & $0,09^{*}$ \\
\hline Pas 3 : CMdériv $A_{L 1}$ & 0,15 & 0,39 & 0,02 \\
\hline \multicolumn{4}{|c|}{ Prédictives de la CMflex $\mathrm{F}_{\mathrm{L} 2} 3^{\mathrm{e}}$ année $(\mathrm{n}=44)$} \\
\hline Pas 1 : lecture mots $\mathrm{F}_{\mathrm{L} 2}$ & 0,13 & 0,18 & $0,18^{* *}$ \\
\hline Pas 2 : vocabulaire $\mathrm{F}_{\mathrm{L} 2}$ & 0,15 & 0,20 & 0,02 \\
\hline Pas 3 : CMdériv $F_{L 2}$ & $0,37^{*}$ & 0,31 & $0,11^{*}$ \\
\hline Pas 4: CMdériv $\mathrm{A}_{\mathrm{L} 1}$ & 0,05 & 0,31 & 0,00 \\
\hline
\end{tabular}

${ }^{* * *}$ r significatif à $\mathrm{p}<0,001,{ }^{* *} \mathrm{p}<0,01,{ }^{*} \mathrm{p}<0,05,{ }^{* * * *} \mathrm{p}<0,07$.

Tableau 5

Régressions hiérarchiques sur la lecture de mots $\mathrm{F}_{\mathrm{L} 2}$ à chaque niveau.

\begin{tabular}{|c|c|c|c|}
\hline & $\sim$ & $\mathrm{R}^{2}$ & $\vartheta \mathrm{R}^{2}$ \\
\hline \multicolumn{4}{|l|}{$2^{\mathrm{e}}$ année $\mathrm{F}_{\mathrm{L}_{2}}(\mathrm{n}=33)$} \\
\hline Pas 1 : vocabulaire $\mathrm{F}_{\mathrm{L} 2}$ & 0,16 & 14 & $14^{*}$ \\
\hline Pas 2 : Mdériv $F_{L 2}$ & 0,18 & 25 & $0,11^{*}$ \\
\hline Pas $3:$ lecture mots $A_{L 1}$ & $0,41^{*}$ & 43 & $0,18^{* *}$ \\
\hline Pas 4 : Mdériv $A_{\mathrm{L} 1}$ & $0,30^{t}$ & 50 & $0,07^{* * * *}$ \\
\hline \multicolumn{4}{|l|}{$3^{\mathrm{e}}$ année $\mathrm{F}_{\mathrm{L} 2}(\mathrm{n}=44)$} \\
\hline \multicolumn{4}{|l|}{ Modèle 1} \\
\hline Pas 1 : vocabulaire $F_{L 2}$ & $0,40^{* *}$ & 0,26 & $0,26^{* *}$ \\
\hline Pas $2:$ Mdériv $F_{L 2}$ & 0,11 & 0,42 & $0,16^{* *}$ \\
\hline Pas $3:$ Mflex $F_{\mathrm{L} 2}$ & 0,07 & 0,43 & 0,01 \\
\hline Pas $4:$ lecture mots $A_{L 1}$ & $0,41^{* * *}$ & 0,59 & $0,16^{* * *}$ \\
\hline Pas 5 : Mdériv $A_{L 1}$ & $0,25^{*}$ & 0,64 & $0,05^{*}$ \\
\hline \multicolumn{4}{|l|}{ Modèle 2} \\
\hline Pas $2:$ Mflex $F_{L 2}$ & 0,07 & 0,34 & $0,08^{*}$ \\
\hline Pas 3 : Mdériv $F_{\mathrm{L} 2}$ & 0,11 & 0,43 & $0,09^{*}$ \\
\hline Pas $4:$ lecture mots $A_{L 1}$ & $0,41^{* * *}$ & 0,59 & $0,16^{* * *}$ \\
\hline Pas $5:$ Mflex $A_{L 1}$ & $-0,08$ & 0,60 & 0,01 \\
\hline Pas 6 : Mdériv $A_{L 1}$ & $0,25^{*}$ & 0,65 & $0,05^{*}$ \\
\hline
\end{tabular}

dépendante comprend en pas 2, le vocabulaire $\mathrm{F}_{\mathrm{L} 2}$, puis en pas 3, la CMdériv $\mathrm{F}_{\mathrm{L} 2}$; Les scores CMflex $\mathrm{F}_{\mathrm{L} 2}$ n'étant pas corrélés avec les scores CMflex $\mathrm{A}_{\mathrm{L} 1}$ (cf. Tableau 2), cette dernière variable n'a pas été introduite dans l'analyse de régression.

En $2^{\mathrm{e}}$ année, les scores CMdériv $\mathrm{A}_{\mathrm{L} 1}$ prédisent une part significative de la variance des scores $\mathrm{CMdériv}_{\mathrm{L} 2}(12 \%, \mathrm{p}<0,05)$ et s'avère même être le seul prédicteur significatif $\left({ }^{\sim}=0,40\right)$. En $3^{\mathrm{e}}$ année, sa contribution n'est en revanche pas significative. À ce niveau, les scores CMflex et CMdériv $\mathrm{F}_{\mathrm{L} 2}$ apportent réciproquement la meilleure contribution l'une sur l'autre ( $~ s=0,33$ et 0,37 respectivement).

3.4.2. Régressions hiérarchiques sur le niveau de lecture de mots $F_{\mathrm{L} 2}$

Afin d'éprouver l'existence d'un transfert de la $\mathrm{CM} \mathrm{A}_{\mathrm{L} 1}$ sur la lecture de mots $\mathrm{F}_{\mathrm{L} 2}$ indépendamment des connaissances lexicales et morphologiques développées dans la langue cible, une série de régressions hiérarchiques a été menée avec, comme variable dépendante, la lecture de mots $\mathrm{F}_{\mathrm{L} 2}$ (cf. Tableau 5). Ces analyses ont été menées séparément à chaque niveau scolaire. En $1^{\mathrm{e}}$ année $\mathrm{F}_{\mathrm{L} 2}$, la lecture de mots $\mathrm{F}_{\mathrm{L} 2}$ n'étant corrélée qu'avec la lecture de mots $\mathrm{A}_{\mathrm{L} 1}(\mathrm{r}=0,64)$ il n'a pas été nécessaire de réaliser une analyse de régression. En $2^{\mathrm{e}}$ et $3^{\mathrm{e}}$ année, les variables mesurées en $\mathrm{F}_{\mathrm{L} 2}$ ont été introduites en premier: le vocabulaire, puis les performances morphologiques disponibles au niveau considéré. 
Ensuite, la lecture de mots $A_{L 1}$ puis les mesures de CM disponibles en L1 ont été entrées dans les modèles de régression ${ }^{13}$.

En $2^{\mathrm{e}}$ année, la lecture de mots $\mathrm{A}_{\mathrm{L} 1}$, introduite au pas 3, prédit une part significative de la variance en lecture de mots $\mathrm{F}_{\mathrm{L} 2}(18 \%, \mathrm{p}<0,05)$. Au pas suivant, la contribution des scores CMdériv $\mathrm{A}_{\mathrm{L} 1}$ n'est que tendancielle $(7 \% \text { de variance expliquée, } p=0,065)^{14}$. En $3^{\mathrm{e}}$ année, toutes les mesures morphologiques sont fiables. Cependant, celles de la $\mathrm{CMflex} \mathrm{A}_{\mathrm{L} 1}$ ne corrèlent pas avec la lecture de mots $\mathrm{F}_{\mathrm{L} 2}$ (cf. Tableau 2) mais corrèlent avec la CMdériv $A_{L 1}$. Deux modèles de régression ont donc été menés pour tester la contribution des scores CMdériv $A_{\mathrm{L} 1}$ après ou non avoir supprimé la part de variance qu'ils partagent avec ceux de CMflex AL1 (non entrés dans l'analyse-modèle 1 -ou entrés en pas 6 modèle 2). Dans le modèle 1, les scores $\mathrm{CMdériv}_{\mathrm{L} 22}$ sont entrés avant les scores $\mathrm{CMflex}_{\mathrm{L} 22}$ (pas 2) et après ces derniers dans le modèle 2 (pas 3). Il en ressort que la contribution des scores CMflex $\mathrm{F}_{\mathrm{L} 2}$ n'est pas significative (cf. modèle 1) alors que celle des scores CMdériv l'est ( $9 \%$ de variance expliquée, cf. modèle 2). En outre, la lecture de mots $A_{\mathrm{L} 1}$ explique $16 \%$ de la variance en lecture de mots $\mathrm{F}_{\mathrm{L} 2}(\mathrm{p}<0,001)$ et les scores CMdériv $\mathrm{A}_{\mathrm{L} 1} 5 \%(\mathrm{p}<0,05)$ quel que soit le modèle. Enfin, parmi les variables introduites, le meilleur prédicteur de la lecture $\mathrm{F}_{\mathrm{L} 2}$ est, aux trois niveaux, la lecture de mots $\mathrm{A}_{\mathrm{L} 1}\left({ }_{\mathrm{s}}=0,41\right.$ à 0,64$)$, suivie, en $3^{e}$ année $\mathrm{F}_{\mathrm{L} 2}$ du vocabulaire $\mathrm{F}_{\mathrm{L} 2}(\backsim=0,40)$ puis des mesures de CMdériv $\mathrm{A}_{\mathrm{L} 1}(\backsim=0,25)$.

\section{Discussion}

L'objectif principal de cette étude était de déterminer dans quelle mesure la CM en L1 contribue à la $\mathrm{CM}$ et à la lecture en $\mathrm{F}_{\mathrm{L} 2}$. Nous tenterons, malgré le manque de fiabilité de certaines mesures sur lequel nous reviendrons en conclusion, de faire ressortir des éléments de réflexions pour les recherches à venir.

Nous voulions tout d'abord mettre en évidence l'existence d'un transfert de la CM entre l' $A_{\mathrm{L} 1}$ et le $\mathrm{F}_{\mathrm{L} 2}$ à différents niveaux d'apprentissage. Pour se faire, nous avons administré à des élèves arabophones en $1^{\mathrm{e}}, 2^{\mathrm{e}}$ et $3^{\mathrm{e}}$ années $\mathrm{F}_{\mathrm{L} 2}$ des tâches morphologiques similaires en $\mathrm{A}_{\mathrm{L} 1}$ et $\mathrm{F}_{\mathrm{L} 2}$. Les résultats ne nous ont pas permis d'étudier ce transfert en $1^{\mathrm{e}}$ année du fait de réponses ne se différenciant pas significativement du hasard. De tels résultats pourraient être mis en lien avec le fait qu'en $1^{\mathrm{e}}$ année, les connaissances lexicales en français sont encore faibles (51\% de bonnes réponses). En $2^{\mathrm{e}}$ année, cette analyse a pu être menée mais seulement sur la CMdériv. À ce niveau, la CMdériv mesurée en $\mathrm{A}_{\mathrm{L} 1}$ semble apporter une contribution non négligeable à ces mêmes compétences en français L2 $(12 \%$ de variance expliquée) après avoir contrôlé les effets des habiletés en lecture. Ainsi, même entre des L1 et L2 aussi éloignées morphologiquement que l'arabe et le français, le transfert interlangue semblerait pouvoir s'effectuer.

Ces résultats complètent ceux de Schiff et Calif (2007) sur l'hébreu L1 et l'E $E_{L 2}$ qui avaient vu apparaître en $4^{\mathrm{e}}$ année d'apprentissage d' $\mathrm{E}_{\mathrm{L} 2}$, une relation interlangue de la $\mathrm{CM}$. Cependant, leurs tâches ne portaient que sur des structures linéaires qui minimisaient les différences interlangues. Nos résultats suggèrent que le transfert pourrait concerner des structures morphologiques n'ayant pas les mêmes principes de construction. Ce transfert apparaîtrait également plus tôt que dans l'étude de Schiff et Calif (2007). Toutefois en $3^{e}$ année, cette contribution de la CMdériv en $A_{L 1}$ sur leur homologue en $F_{L 2}$ n'est toujours pas significative, la CMflex puis la lecture en $\mathrm{F}_{\mathrm{L} 2}$ apparaissant comme les meilleurs prédicteurs de la CMdériv dans la même langue. Il semble donc qu'en $3^{e}$ année de $F_{L 2}$, le poids des CM dans la même langue mais sur une autre dimension puissent prendre le pas sur le celui des compétences en L1. Cette observation suggère une plus grande autonomie des connaissances Mdériv en $F_{L 2}$. Le transfert de la CMdériv d'une L1 dont la structure morphologique est non linéaire vers une L2 morphologiquement linéaire pourrait ainsi s'effectuer avant même que le système écrit de la L2 soit relativement bien maîtrisé.

Ainsi, la maîtrise croissante des compétences en lecture pourrait expliquer la disparition du transfert de la CMdériv $A_{L 1}$ ce qui irait dans le sens de 1'hypothèse de Bialystoket al. (2005). Avec l'avancée

\footnotetext{
13 La conscience phonologique n'a pas été entrées dans ces analyses car aux niveaux scolaires étudiés, il est probable que son rôle soit atténué (Singson, Mahony, \& Mann, 2000).

14 Dans ce modèle, les mesures $\mathrm{CMflex}_{\mathrm{L} 2}$ et $\mathrm{A}_{\mathrm{L} 1}$ n'ont pas pu être introduites.
} 
dans les apprentissages, les apprenants, dès la $3^{\mathrm{e}}$ année de L2, pourraient mettre en place des traitements morphologiques moins dépendants de leur L1 car ils maîtriseraient davantage le système écrit du français (les scores en lecture de mots $\mathrm{F}_{\mathrm{L} 2}$ apparaissent d'ailleurs significativement supérieurs en $3^{\mathrm{e}}$ année comparativement à la $2^{\mathrm{e}}$ année). En étant de plus en plus confrontés aux mots écrits français, leurs traitements morphologiques pourraient se "spécialiser » et rendre ainsi plus saillantes les relations fonctionnelles et structurales entre les caractéristiques flexionnelles et dérivationnelles du français. En revanche, comme le suggèrent les résultats de Ramirez et al. (2010) ou de Schiff et Calif (2007), le lien L1/L2 pourrait être encore présent après plusieurs années d'apprentissage lorsque les structures morphologiques sont similaires. Ce postulat n'a toutefois pas pu être vérifié par l'observation d'un transfert de la CMflex de l' $\mathrm{A}_{\mathrm{L} 1}$ sur le $\mathrm{F}_{\mathrm{L} 2}$. Ce transfert n'a pu être étudié qu'en $3^{\mathrm{e}}$ année et les résultats ne semblent pas mettre en évidence une corrélation significative entre $\mathrm{CMflex}_{\mathrm{L} 1}$ et $\mathrm{CMflex}_{\mathrm{L} 2}$. En revanche, la $\mathrm{CMdériv}_{\mathrm{L} 2}$ apparaitrait, à ce niveau, comme le meilleur prédicteur de la $\mathrm{CMflex} \mathrm{F}_{\mathrm{L} 2}$, étant elle-même le meilleur prédicteur de la CMdériv $\mathrm{F}_{\mathrm{L} 2}$. Cette contribution réciproque inviterait à considérer les similitudes entre les traitements des aspects flexionnels et dérivationnels en francais. Il faut cependant considérer les interprétations des résultats concernant la CMflex $F_{\mathrm{L} 2}$ avec précaution, d'une part, car elle n'a pu faire l'objet d'analyse que sur un seul niveau scolaire et, d'autre part, car la fiabilité de cette mesure est faible. Ce manque de consistance peut d'ailleurs être à l'origine du manque de résultats significatifs sur cette épreuve en particulier. Malgré cette limite, il est cohérent d'envisager que les habiletés en $\mathrm{F}_{\mathrm{L} 2}$ soient à ce niveau scolaire suffisamment développées pour être indépendantes des habiletés en L1.

Le second objectif concernait le transfert interlangue de la CM en L1 sur la lecture de mots en $\mathrm{F}_{\mathrm{L} 2}$. Ce n'est qu'au dernier niveau étudié $\left(3^{\mathrm{e}}\right.$ année $\mathrm{F}_{\mathrm{L} 2}$ ) qu'indépendamment du vocabulaire, de la $\mathrm{CM} \mathrm{F}_{\mathrm{L} 2}$ et de la lecture de mots $A_{L 1}$, la CMdériv $A_{L 1}$ contribuerait à la lecture de mots $F_{L 2}$. Ce résultat appuie l'hypothèse sur le rôle des connaissances morphologiques les plus déterminantes pour lire en arabe L1 (CMdériv) dans la lecture en $\mathrm{F}_{\mathrm{L} 2}$, ceci en dépit des différences structurales de la morphologie entre ces deux langues. Toutefois, ce transfert interviendrait relativement tardivement. Il se pourrait que, $\mathrm{du}$ fait de la distance orthographique entre 1'arabe et le français, les apprentis lecteurs en $\mathrm{F}_{\mathrm{L} 2}$ soient surtout focalisés en début d'apprentissage sur le décodage graphophonologique, ce qui laisserait peu de place pour des traitements de nature sémantique comme ceux de la morphologie. Une fois le décodage installé, la mise en place d'une analyse de la signification des mots entiers expliquerait l'intervention du vocabulaire dès la $2^{\mathrm{e}}$ année. L'analyse des unités morphémiques issue des acquisitions en L1 pourrait alors intervenir dans un troisième temps. Cette intervention semble toutefois se limiter à la CMdériv. Le transfert de la CMdériv pourrait être plus précoce du fait de l'importance, de la richesse et de la productivité de cette dimension en arabe standard. Ces résultats rappellent d'ailleurs ceux d'Abu-Rabia (2007) en arabe qui n'avait pas observé le rôle des compétences Mflex lors de la lecture.

L'apparition d'un transfert de la CMdériv de l'A $\mathrm{L}_{\mathrm{L} 1}$ vers le $\mathrm{F}_{\mathrm{L} 2} \mathrm{n}$ 'est pas en accord avec l'hypothèse de la transparence morphologique posée par Saiegh-Haddad et Geva (2008). En se basant sur leurs résultats, les auteurs avancent que les processus morphologiques seraient davantage utilisés dans les langues morphologiquement transparentes qu'opaques. Ils n'ont, en effet, pas observé de corrélation interlangue sur la CMdériv, ni de contribution de la CMdériv en anglais L1 sur la lecture en arabe L2. $\mathrm{Au}$ contraire, nos résultats suggèrent que le transfert morphologique d'une L1 morphologiquement riche pourrait être facilité même si cette morphologie est peu transparente. Comparativement à la CMflex, la CMdériv pourrait relever d'une dimension particulièrement importante pour des apprenants arabophones car la structure racine/schème est particulièrement accentuée et productive en arabe ce qui pourrait faciliter son transfert. Ainsi, il semble que le transfert de la CMdériv ne soit pas seulement dépendant du degré de transparence de la morphologie et de ses aspects topologiques mais dépende surtout de la richesse et de l'importance de cette dimension pour lire en L1.

Nos résultats appuient la définition du transfert telle que proposée par Tardif (1999) ${ }^{15}$ en suggérant la mise en place de compétences transversales à traiter les aspects morphologiques relevant de processus centraux, en particulier pour ce qui concerne les dimensions les plus prégnantes en L1. Une fois mises en place, ces compétences pourraient être mobilisées lors de l'apprentissage d'une

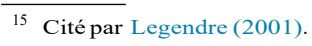


L2. Pour ce qui concerne le développement morphologique, il se pourrait qu'après quelques années d'apprentissage, la prise en compte des aspects spécifiques à la L2 puissent venir compléter les processus centraux initiaux. Ces interprétations doivent toutefois être validées par d'autres recherches du fait de la faiblesse des \& de Cronbach obtenus dans cette étude.

Avant d'envisager le transfert interlangue, nous avions tout d'abord vérifié l'effet du niveau scolaire sur le vocabulaire $\mathrm{F}_{\mathrm{L} 2}$, la lecture de mots $\mathrm{F}_{\mathrm{L} 2}$ et $\mathrm{A}_{\mathrm{L} 1}$, et sur une partie des mesures de CMdériv $\mathrm{A}_{\mathrm{L} 1}$ et $\mathrm{F}_{\mathrm{L} 2}$. Si les performances augmentent avec le niveau scolaire pour le vocabulaire en français et la lecture dans les deux langues, les résultats sont plus difficiles à interpréter pour la CM. En effet, les résultats apparaissent supérieurs au niveau du hasard à partir de la $1^{\mathrm{e}}$ année pour la CMdériv $\mathrm{A}_{\mathrm{L} 1}$ ou de la $2^{\mathrm{e}}$ année pour la CMdériv $\mathrm{F}_{\mathrm{L} 2}$ mais seulement en $3^{\mathrm{e}}$ année pour la $\mathrm{CMflex}{ }^{16}$, bien que la différence entre CMdériv et CMflex à ce niveau scolaire, en $A_{L 1}$ comme en $F_{L 2}$, ne soit pas significative. Ainsi, nos analyses ne confirment pas la plus grande précocité du développement de la CMflex comparativement à la CMdériv observées dans d'autres langues (Kuo \& Anderson, 2006). Bien qu'il ait été vérifié, dans les deux langues, que les mots proposés ne soient pas plus fréquents dans la tâche CMdériv que CMflex, il est possible que les fréquences des schèmes ou affixes choisis ne soient pas équivalentes entre les deux tâches. Il se pourrait aussi qu'il y ait une spécificité des apprenants arabophones ou une singularité de l'enseignement dans un pays arabophone comme la Tunisie. Nous pourrions faire l'hypothèse que l'enseignement orienterait les apprentissages vers les aspects morpho-dérivationnels qui se révèlent déterminants en arabe, du fait de la structure en racine/schème particulièrement productive.

\section{Conclusion}

L'intérêt d'étudier le transfert entre l' $\mathrm{A}_{\mathrm{L} 1}$ et le $\mathrm{F}_{\mathrm{L} 2}$ repose sur l'éloignement de ces langues tant au niveau orthographique que morphologique. La richesse de la morphologie de l'arabe et son importance pour la lecture, de même que la coexistence de structures linéaires et non linéaires dans cette langue, sont des caractéristiques intéressantes pour identifier l'intervention alternée ou simultanée des processus centraux et spécifiques aux langues. L'existence de compétences transversales à traiter les aspects morphologiques des mots même structuralement très éloignés et de pouvoir faire appel à ces compétences lors de la lecture en $\mathrm{F}_{\mathrm{L} 2}$ interroge sur leur nature. De notre point de vue, les processus centraux impliqués lors du transfert morphologique pourraient rendre compte d'habiletés segmentales sublexicales ou encore d'habiletés à traiter les informations phonologiques, orthographiques et sémantiques de façon coordonnée (Saiegh-Haddad \& Geva, 2008). Il convient de souligner que nos résultats suggèrent aussi que l'intervention de processus centraux est insuffisante pour saisir l'ensemble des mécanismes présents au cours de l'avancée dans les apprentissages en L2. Il est ainsi indispensable de mener une recherche de plus grande ampleur distinguant ces différentes habiletés pour rendre compte de leur implication, relevant ou non de processus centraux.

Cette dynamique du transfert ne manquera pas d'alimenter les réflexions des enseignants afin de tenir compte de la façon et du contexte dans lequel les élèves peuvent mobiliser des ressources et outils cognitifs leur permettant de réactualiser leurs connaissances (Tardif, $1999^{17}$ ).

Cependant, cette recherche n'est pas exempte de toutes fragilités notamment méthodologiques. La faiblesse des coefficients de cohérence interne sur plusieurs tâches constitue une limite importante de la fiabilité des mesures effectuées et donc des résultats, interprétations et implications qui peuvent en émerger. Cette limite pose également la question de l'utilisation des tâches de détection d'intrus et les processus qu'elles impliquent, en particulier pour mesurer des connaissances dont on sait qu'elles sont acquises relativement précocement (e.g. CMflex). Il est possible que la détection d'une forme non fléchie partageant des apparences phonologiques et orthographiques avec une forme fléchie très régulière sollicite plus ou moins fortement les fonctions exécutives, ce qui pourrait être important pour comprendre les mécanismes sous-jacents à la $\mathrm{CM}$ et les mesurer. D'autre part, pour mesurer les

\footnotetext{
16 Parce que les \& de Cronbach augmentent de façon importante lorsque l'analyse porte essentiellement sur les participants de $3^{\mathrm{e}}$ année (passant de 0,61 à 0,70 pour la $C M f l e x A_{L 1}$ et de 0,39 à 0,52 pour la CMflex $F_{L 2}$ ) nous considérerons qu'àce niveau scolaire, ces tâches permettent effectivement de mesurer la CMflex.

17 Cité par Legendre (2001).
} 
habiletés de CMflex de facon consistante, il importe de ne faire porter la tâche que sur une seule règle morphologique à la fois (e.g. la conjugaison verbale) et non sur plusieurs et d'inclure un nombre suffisant d'items. Les recherches ultérieures devront ainsi tenir compte de ces constats pour améliorer la fiabilité des résultats et ainsi, la contribution de données empiriques interlangues à la compréhension du transfert.

\section{Déclaration de liens d'intérêts}

Les auteurs déclarent ne pas avoir de liens d'intérêts.

\section{Références non citées}

McBride-Chang, Wagner, Muse, Chow, \& Shu, 2005 and Pasquarella, Chen, Lam, Luo, \& Ramirez, 2011

\section{Remerciements}

Cette recherche a fait l'objet d'une subvention de l'AUF.

\section{Annexe 1. Exemples d'items aux tâches CM}

Tâche CMflex $\mathrm{AL}_{1}$ :

Exemple d'item sur l'inaccompli : quel est le verbe qui n'est pas conjugué comme :

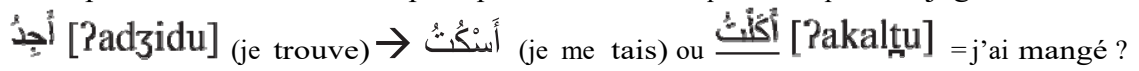

Exemple d'item sur le genre : quel est le nom qui n'est pas un féminin comme

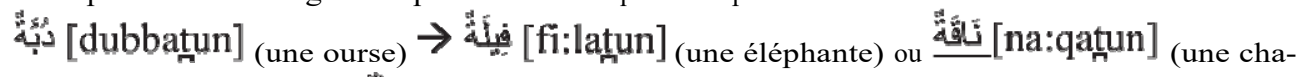

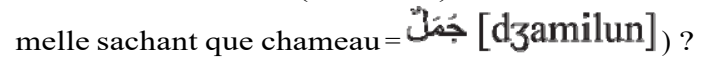

Tâche CMderiv $\mathrm{AL}_{1}$ :

Exemple d'item sur les substantifs : quel est le mot qui n'a pas de racine comme

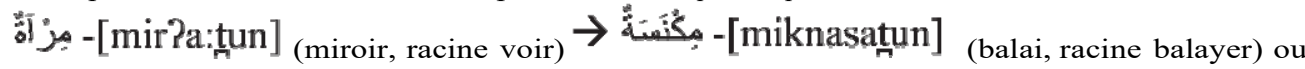

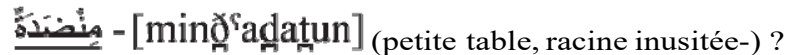

Exemple d'item sur les verbes: quel est le mot qui n'a pas de racine comme

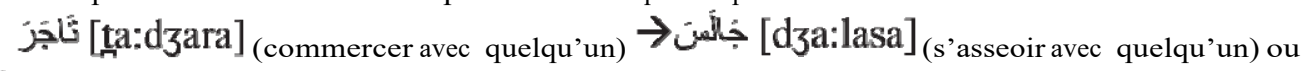
عَ [ra:ladza] (soigner, guérir)

Tâche CMflex $\mathrm{FL}_{2}$ :

Exemple d'item sur le présent de l'indicatif: quel est le verbe qui n'est pas conjugué comme nous laissons $\rightarrow$ nous tirons ou nous boirons ?

Exemple d'item sur le genre : quel est le nom qui n'est pas un féminin comme forte $\rightarrow$ cuite ou triste?

Tâche CMderiv $\mathrm{FL}_{2}$ :

Exemple d'item préfixé : quel est le mot qui ne vient pas d'un autre mot comme déboucher $\rightarrow$ déplacer ou décider ?

Exemple d'item suffixé : quel est le mot qui ne vient pas d'un autre mot comme cendrier $\rightarrow$ boîtier ou cahier?

\section{Références}

Abu Hammad, H., Ibrahim, R., \& Share, D. L. (2014). Cognitive predictors of early reading ability in Arabic: A longitudinal study from kindergarten to grade 2. In E. Saiegh-Haddad, \& R. M. Joshi (Eds.), Handbook of Arabic literacy: Insights and perspectives (pp. 171-194). New York: Springer Dordrecht. 
Abu-Rabia, S. (2007). The role of morphology and short vowelization in reading Arabic among normal and dyslexic readers in grades 3, 6, 9, and 12. Journal of Psycholinguist Research, 36, 89-106.

Al Ghanem, R., \& Kearns, D. M. (2015). Orthographic, phonological, and morphological skills and children's word reading in Arabic: A literature review. Reading Research Quarterly, 50(1), 83-109.

Ammar, M. (2002). L'assemblage phonologique : Sa nature et son fonctionnement chez des enfants lecteurs en arabe. Enfance, $54(2), 155-163$.

Berninger, V. W., Abott, R. D., Nagy, W., \& Carlisle, J. (2010). Growth in phonological, orthographic, and morphological awareness in grades 1 to 6. Journal of Psycholinguistic Research, 39(2), 141-163.

Bialystok, E., McBride-Chang, C., \& Luk, G. (2005). Bilingualism, language proficiency, and learning to read in two writing systems. Journal of Educational Psychology, 97(4), 580-590.

Boudelaa, S. (2014). Is the Arabic mental lexicon morpheme-based or stem-based? Implications for spoken and written word recognition. In E. Saiegh-Haddad, \& R. M. Joshi (Eds.), Handbook of Arabic literacy: Insights and perspectives (pp. 31-54). New York: Springer Dordrecht.

Boukadida, N., Gombert, J. E., \& Maaouia, A. (2009). Contribution des connaissances morphologiques à l'apprentissage de la lecture en arabe. In N. Marec-Breton, A.-S. Besse, F. de la Haye, N. Bonneton, \& E. Bonjour (Eds.), L'apprentissage de la langue écrite : Approche cognitive (pp. 107-118). Rennes: PUR.

Carlisle, J. F. (1995). Morphological awareness and early reading achievement. In L. B. Feldman (Ed.), Morphological aspects of language processing (pp. 189-209). Hillsdale, NJ, England: Lawrence Erlbaum Associates.

Carlisle, J. F. (2003). Morphology matters in learning to read: A commentary. Reading Psychology, 24, 291-322.

Carlisle, J., McBride-Chang, C., Nagy, W., \& Nunes, T. (2010). Effects of instruction in morphological awareness on literacy achievement: An integrative review. Reading Research Quarterly, 45(4), 464-487.

Casalis, S., Quémart, P., \& Duncan, L. G. (2015). How language affects children's use of derivational morphology in visual word and pseudoword processing: Evidence from a cross-language study. Frontiers in Psychology, http://dx.doi.org/10.3389/fpsyg.2015.00452

Casalis, S., \& Louis-Alexandre, M.-F. (2000). Morphological analysis, phonological analysis and learning to read French: A longitudinal study. Reading \& Writing, 12(3), 303-335.

Colé, P., Royer, C., Leuwers, C., \& Casalis, S. (2004). Les connaissances morphologiques dérivationnelles et l'apprentissage de la lecture chez l'apprenti-lecteur français du CP au CE2. L'année psychologique, 104(4), 701-750.

Cummins, J. (2000). Language, power and pedagogy; Bilingual children in the crossfire. Philadelphia: Multilingual matters.

Deacon, H., Wade-Woolley, L., \& Kirby, J. (2007). Crossover: The role of morphological awareness in French immersion children's reading. Developmental Psychology, 43(3), 732-746.

Deltour, J.-J., \& Hupkens, D. (1980). Test de vocabulaire actif et passif pour enfants de 3 à 5 ans. Liège: Éditions scientifiques et psychologiques.

Genesee, F., Geva, E., Dressler, C., \& Kamil, M. L. (2008). Cross-linguistic relationships in second-language learners. In D. August, $\&$ T. Shanahan (Eds.), Developing reading and writing in second-language learners (pp. 61-93). New York: Routledge.

Geva, E., \& Siegel, L. S. (2000). Orthographic and cognitive factors in the concurrent development of basic reading skills in two languages. Reading \& Writing, 12(1), 1-30.

Gleitman, L. R. (1985). Orthographic resources affect reading acquisition if they are used. RAE, 6, 24-36.

Hipfner-Boucher, K., Lam, K., Chen, X., \& Deacon, S. H. (2015). Exploring the effects of word features on French immersion children's ability to deconstruct morphologically complex words. Writing Systems Research, 7(2), 157-168.

Howel, D. C. (1998). Méthodes statistiques en sciences humaines. Bruxelles: De Boeck Université.

Kahn-Horwitz, J., Shimron, J., \& Sparks, R. L. (2005). Predicting foreign language reading achievement in elementary school students. Reading \& Writing, 18(6), 527-558.

Ke, S., \& Xiao, F. (2015). Cross-linguistic transfer of morphological awareness between Chinese and English. Language Awareness, 4, 355-380.

Khomsi, A. (1999). Lecture de mots et compréhension-Révisée. Tâche d'évaluation de la compétence en lecture. Paris: ECPA.

Kieffer, M. J., Biancarosa, G., \& Mancilla-Martinez, J. (2013). Roles of morphological awareness in the reading comprehension of Spanish-speaking language minority learners: Exploring partial mediation by vocabulary and reading fluency. Applied Psycholinguistics, 34(4), 697-725.

Kim, Y.-S. (2009). Crosslinguistic influence on phonological awareness for Korean-English bilingual children. Reading \& Writing, 22, 843-861.

Kirby, J. R., Deacon, S. H., Bowers, P. N., Izenberg, L., Wade-Woolley, L., \& Parrila, R. (2012). Children's morphological awareness and reading ability. Reading \& Writing, 25, 389-410.

Koda, K. (2008). Impacts of prior literacy experience on second language learning to read. In K. Koda, \& A. M. Zehler (Eds.), Learning to read across languages cross-linguistic relationships in first- and second-language literacy development (pp. 68-96). NY: Routledge.

Kuo, L.-J., \& Anderson, R. C. (2006). Morphological awareness and learning to read: A cross-language perspective. Educational Psychologist, 41(3), 161-180.

Kuo, L.-J., \& Anderson, R. C. (2010). Beyond cross-language transfer: Reconceptualizing the impact of early bilingualism on phonological awareness. Scientific Studies of Reading, 14(4), 365-385.

Legendre, M. F. (2001). Le transfert des apprentissages. Montréal: Les éditions Logiques. Revue des sciences de l'éducation, 272 , 441-444 (Tardiff, J., 1999).

Lété, B., Sprenger-Charolles, L., \& Colé, P. (2004). Manulex: A grade-level lexical database from French elementary-school readers. Behavior Research Methods, Instruments, \& Computers, 36, 156-166.

Levesque, K. C., Kieffer, M. J., \& Deacon, S. H. (2017). Morphological awareness and reading comprehension: Examining mediating factors. Journal of Experimental Child Psychology, 160, 1-20.

Marec-Breton, N., \& Gombert, J. E. (2007). How young children read complex words? Educacao, 4, 49-80.

McBride-Chang, C., Wagner, R. K., Muse, A., Chow, B. W.-Y., \& Shu, H. (2005). The role of morphological awareness in children's vocabulary acquisition in English. Applied Psycholinguistics, 26(3), 415-435. 
Pasquarella, A., Chen, X., Lam, K., Luo, Y., \& Ramirez, G. (2011). Cross-language transfert of morphological awareness in ChineseEnglish bilinguals. Journal of Research in Reading, 34(1), 23-42.

Ramirez, G., Chen, X., Geva, E., \& Kiefer, H. (2010). Morphological awareness in Spanish-Speaking english language learners: Within and cross-language effects on word reading. Reading \& Writing, 23, 337-358.

Ramirez, G., Chen, X., Geva, E., \& Luo, Y. (2011). Morphological awareness and word reading in English language learners: Evidence from Spanish- and Chinese-speaking children. Applied Psycholinguistics, 32, 601-618.

Ramirez, G., Chen, X., \& Pasquarella, A. (2013). Cross-linguistic transfer of morphological awareness in Spanish-speaking ELLs: The facilitating effect of cognate knowledge. Topics in Language Disorders, 33, 73-92.

Saiegh-Haddad, E., \& Henkin-Roitfarb, R. (2014). The structure of Arabic language and orthography. In E. Saiegh-Haddad, \& R. M. Joshi (Eds.), Handbook of Arabic literacy: Insights and perspectives (pp. 3-28). NY: Springer Dordrecht.

Saiegh-Haddad, E., \& Geva, E. (2008). Morphological awareness, phonological awareness and reading in English-Arabic bilingual children. Reading \& Writing, 21, 481-504.

Sanchez, M., Ecalle, J., \& Magnan, A. (2012). L'influence précoce des connaissances morphologiques et orthographiques sur l'apprentissage de la lecture. Une étude longitudinale de la GSM au CE1. Psychologie française, 57(4), 277-290.

Schiff, R., \& Calif, S. (2007). Role of phonological and morphological awareness in L2 oral word reading. Language Learning, 57(2), 271-298.

Singson, M., Mahony, D., \& Mann, V. (2000). The relation between reading ability and morphological skills: Evidence from derivation suffixes. Reading \& Writing, 12(3), 219-252.

Wang, M., Cheng, C., \& Chen, S. W. (2006). Contribution of morphological awareness to Chinese-English biliteracy acquisition. Journal of Educational Psychology, 98, 542-553.

Wang, M., Ko, I., \& Choi, J. (2009). The importance of morphological awareness in Korean-English biliteracy acquisition. Contemporary Educational Psychology, 34, 132-142.

Zhang, D. (2013). Linguistic distance effect on cross-linguistic transfer of morphological awareness. Applied Psycholinguistics, $34,917-942$.

Zhang, H. S. (2014). Morphological awareness in vocabulary acquisition among Chinese-speaking children: testing partial mediation via lexical inference ability. Reading Research Quarterly, 50(1), 129-142.

Zhang, D., Koda, K., \& Leong, C. K. (2016). Morphological awareness and bilingual word learning: A longitudinal Structural Equation Modeling study. Reading \& Writing, 29, 383-407.

Ziegler, J. C., Bertrand, D., Tóth, D., Csépe, V., Reis, A., et al. (2010). Orthographic depth and its impact on universal predictors of reading: A cross-language investigation. Psychological Science, 21, 551-559. 